\title{
Can KRAS and BRAF mutations limit the benefit of liver resection in metastatic colorectal cancer patients? A systematic review and meta-analysis
}

\author{
Francesco Passigliaa $^{\mathrm{a}, 1}$, Giuseppe Bronte ${ }^{\mathrm{a}, 1}$, Viviana Bazan ${ }^{\mathrm{a}}$, Antonio Galvano ${ }^{\mathrm{a}}$, \\ Bruno Vincenzi ${ }^{\mathrm{b}}$, Antonio Russo ${ }^{\mathrm{a}, *}$ \\ a Department of Surgical, Oncological and Oral Sciences, Section of Medical Oncology, University of Palermo, Palermo, Italy \\ ${ }^{\mathrm{b}}$ Medical Oncology Department, Campus Biomedico, University of Rome, Rome, Italy
}

\section{Contents}

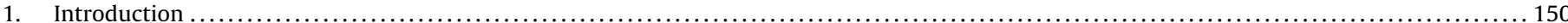

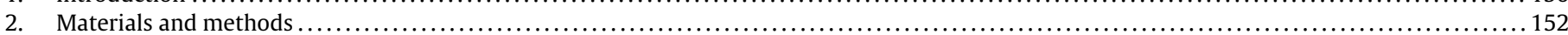

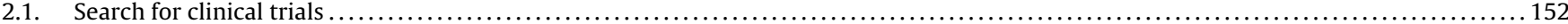

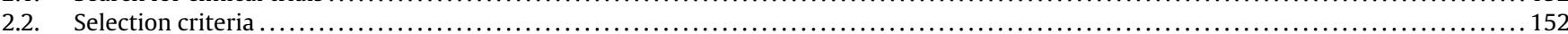

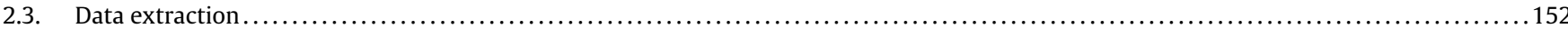

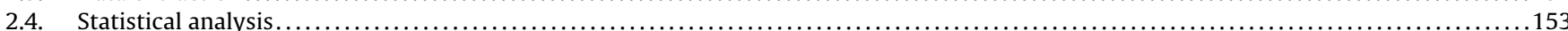

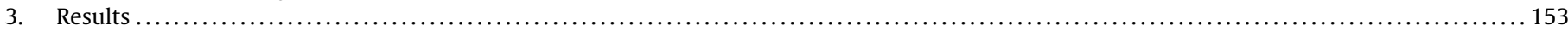

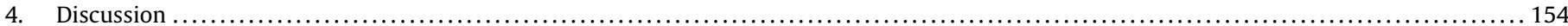

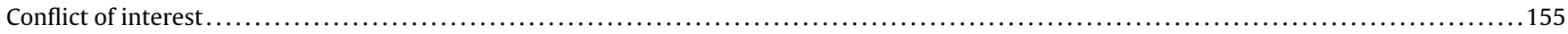

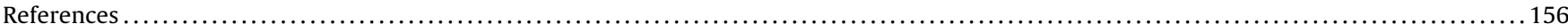

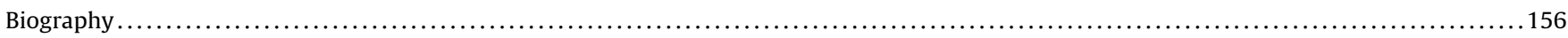

\section{A R T I C L E I N F O}

\section{Article history:}

Received 12 August 2015

Received in revised form 27 October 2015

Accepted 23 December 2015

\section{Keywords:}

KRAS

BRAF

Prognostic biomarker

Liver metastasis

Colorectal cancer

\begin{abstract}
A B S T R A C T
Background: Clinical trials investigated the potential role of both KRAS and BRAF mutations, as prognostic biomarkers, in colorectal cancer (CRC) patients who underwent surgical treatment of CRC-related liver metastases (CLM), showing conflicting results. This meta-analysis aims to review all the studies reporting survival outcomes (recurrence free survival (RFS), and/or overall survival (OS)) of patients undergoing resection of CLM, stratified according to KRAS and/or BRAF mutation status.

Materials and methods: Data from all published studies reporting survival outcomes (RFS and/or OS) of CRC patients who received resection of CLM, stratified by KRAS and/or BRAF mutation status were collected, according to the PRISMA guidelines. Pooled HRs were calculated for both the OS and/or RFS.

Results: Seven eligible trials (1403 patients) were included. Pooled analysis showed that KRAS mutations predicted a significantly worse both RFS (HR: 1.65; 95\% CI: 1.23-2.21) and OS (HR: 1.86; 95\% CI: 1.51-2.30) in patients who underwent surgical resection of CLM. BRAF mutations were also associated with a significantly worse OS (HR: 3.90; 95\% CI: 1.96-7.73) in this subgroup of patients.

Conclusions: This meta-analysis suggests both KRAS and BRAF mutations as poor, prognostic biomarkers, associated with worse survival outcomes, in patients undergoing hepatic resection of CLM.
\end{abstract}

(c) 2016 Elsevier Ireland Ltd. All rights reserved.

\footnotetext{
* Corresponding author at: Medical Oncology Director, Section of Medical Oncology, Department of Surgical, Oncological and Oral Sciences, Palermo University Hospital, Via del Vespro 129, 90127 Palermo, Italy. Fax: +39 916554529.

E-mail address: antonio.russo@usa.net (A. Russo).

${ }^{1}$ These authors equally contributed to this work.
}

\section{Introduction}

The liver is the most common metastatic site (Adam et al., 2009) in colorectal cancer (CRC) patients. Twenty to twenty-five percent $(20-25 \%)$ of patients have clinically detectable colorectal-related liver metastases (CLM) at the initial diagnosis and approximately $50 \%$ of the patients develop CLM during their disease course 
(Garden et al., 2006). Resection of the CLM, sometimes in combination with other local treatment modalities, such as radiofrequency ablation (RFA), has become the standard of care, and offers the only potential for cure (Chiappa et al., 2009; Ksienski et al., 2010). The development of new surgical approaches, together with an increasing use of the perioperative systemic therapy, have led to an increase of the percentage of patients potentially eligible for curative liver resection (Rolfo et al., 2014, 2013; Bronte et al., 2015, 2013). In most series, up to $25 \%$ of patients presenting with stage IV CRC undergo hepatic resection (Kopetz et al., 2009), with reported 5-year survival rates up to 50\% (Taylor et al., 2010; Choti et al., 2002; Chua et al., 2011). However over one-half of patients will develop recurrence within 2 years (de Jong et al., 2009; Fong et al., 1999; Nordlinger et al., 1996), not getting any long-term survival benefit from hepatic resection.

Metastatic colorectal cancer is a very heterogeneous disease, with a strong, both clinical and biological variability, among different patients (Bronte et al., 2014). Therefore difficulties remain in deciding who is a good candidate for liver resection. Many studies have examined several clinical and pathological factors, including number and size of CLM, disease-free interval from primary to CLM, carcinoembryonic antigen (CEA) level, primary tumor stage, synchronous or metachronous CLM, as potential prognostic determinants of survival after surgical resection of CLM, in order to establish a preoperative scoring system that is able to predict the risk of recurrence after resection, and ultimately allows the selection of those patients who may benefit more from surgery (Choti et al., 2002; de Jong et al., 2009; Fong et al., 1999; Nordlinger et al., 1996; Mann et al., 2004; Nagashima et al., 2006a,b).

To date, there are not molecular biomarkers, approved in the clinical setting, that are able to predict such biological differences, favoring the identification of patients, at high risk of relapse (Russo et al., 2009). RAS mutations, including both KRAS and NRAS gene mutations, have been reported in about $50 \%$ of primary CRC, and have been associated with resistance to the anti-epidermal growth factor receptor (EGFR) monoclonal antibodies (MoAbs), Cetuximab

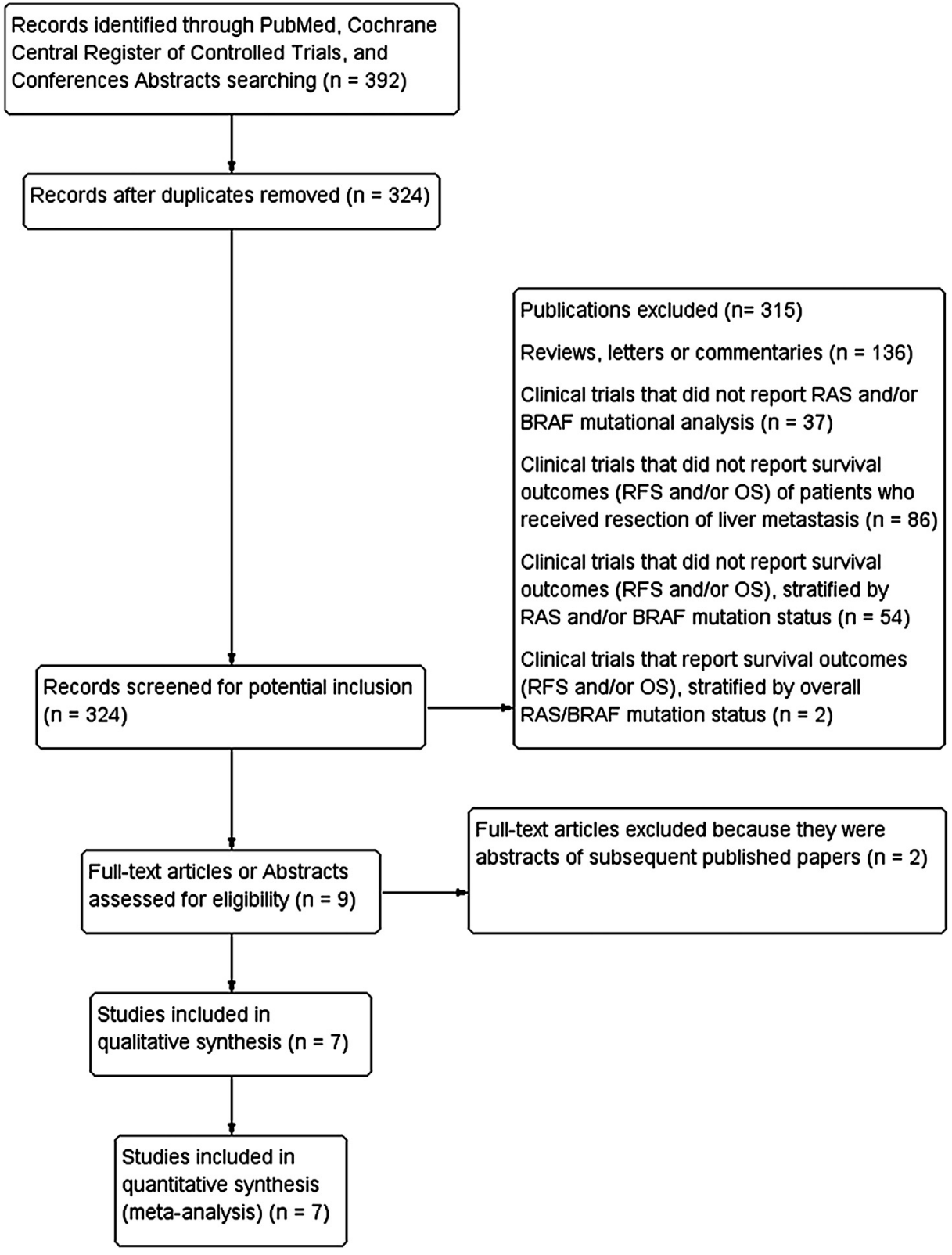

Fig. 1. Flow-chart of studies selection. 


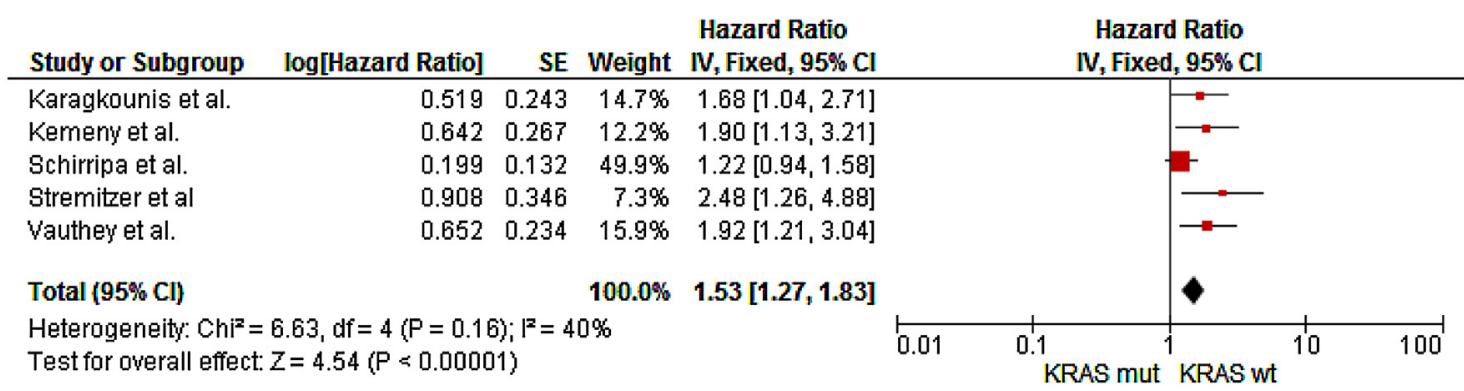

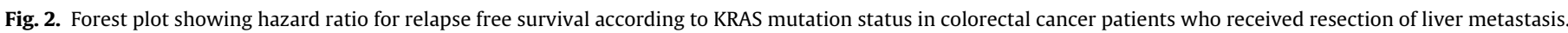

and Panitumumab, in the metastatic disease (Rizzo et al., 2010; Bronte et al., 2011). This has been well exploited by several randomized trials (De Roock et al., 2010; Bokemeyer et al., 2012; Douillard et al., 2013; Heinemann et al., 2014; Schwartzberg et al., 2014; Van Cutsem et al., 2015; Bokemeyer et al., 2015), that have overall shown significant poorer response rates and inferior survival outcomes in RAS-mutated patients receiving chemotherapy plus anti-EGFR MoAbs, compared to RAS-wild type population. RAS mutations represent the only, molecular, predictive biomarker approved for clinical use, while their prognostic role is still debated. Several randomized studies have shown no significant survival differences between KRAS mutated and KRAS wild-type, CRC patients, independently of anti-EGFR therapy (Price et al., 2011; Hecht et al., 2009; Kastrinakis et al., 1995; Russo et al., 1998; Tol et al., 2010), while others have demonstrated a prognostic role of KRAS in advanced disease (Richman et al., 2009; Maughan et al., 2011; Tejpar et al., 2012). BRAF mutations have been described in about $10 \%$ of primary CRC, representing a well-known negative prognostic factor, associated with worse survival outcomes, regardless of any treatment received (Ahn et al., 2014).

Several studies have recently investigated the potential role of KRAS and/or BRAF mutations, as prognostic biomarkers, in patients who underwent surgical treatment of CLM. The majority of such studies have shown that both KRAS and BRAF, respectively detected in about $30 \%$ and $3 \%$ of CLM, are associated with worse survival outcomes of patients undergoing liver resection (Nash et al., 2010; Karagkounis et al., 2013; Vauthey et al., 2013; Stremitzer et al., 2012), while some other studies have not found a significant association (Kemeny et al., 2014; Teng et al., 2012; Schirripa et al., 2015). The aim of this meta-analysis is to combine and analyze simultaneously all the studies reporting the survival outcomes (recurrence free survival (RFS), and/or overall survival (OS)) of patients who received resection of CLM, stratified by KRAS and/or BRAF mutation status, in order to provide a more precise estimation of the prognostic value of both KRAS and BRAF, in patients undergoing surgical therapy for CLM.

\section{Materials and methods}

\subsection{Search for clinical trials}

We performed our meta-analysis according to a predefined written protocol. We searched for all published studies, that report the survival outcomes (RFS and/or OS) of patients who received resection of CLM, stratified by KRAS and/or BRAF mutation status. Publications were identified by an electronic search in Medline, using PubMed online service, updated in May 2015. The search for publications was made by other databases including the Cochrane Library and EMBASE. However the search on PubMed allowed the widest collection of publications about this topic. The following search terms were used: "KRAS", "BRAF", "colorectal cancer", "CRC", "prognostic", "liver metastases", "liver resection" "hepatic surgery”. The search was limited to human studies in the English language. The results were supplemented with manual searches of American Society of Clinical Oncology meeting proceedings, references of selected articles and published reviews. A systematic review on this topic in the Cochrane database of systematic reviews was not found.

\subsection{Selection criteria}

According to this search clinical trials were taken into account if they had to fulfill all the following inclusion criteria: (1) only patients with metastatic colorectal cancer were included; (2) only patients who underwent resection of CLM were included (3) studies that report the survival outcomes (RFS and/or OS) of patients who received resection of CLM; (4) studies that report the survival outcomes (RFS and/or OS), stratified by KRAS and/or BRAF mutation status.

\subsection{Data extraction}

Two authors independently selected studies according to the aforementioned inclusion criteria. If these two authors could not

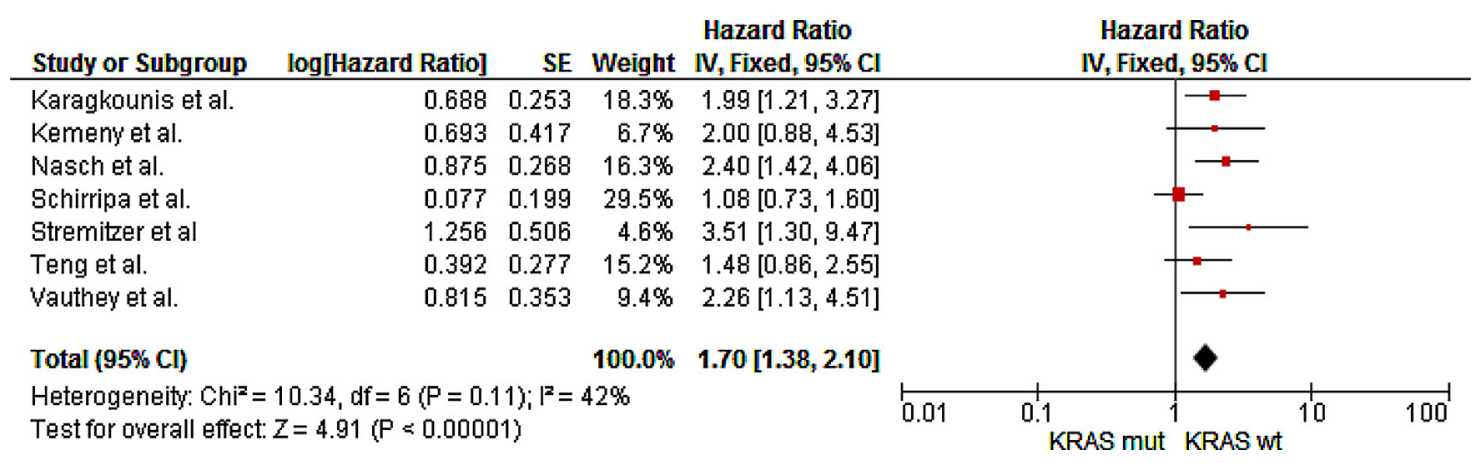

Fig. 3. Forest plot showing hazard ratio for overall survival according to KRAS mutation status in colorectal cancer patients who received resection of liver metastasis. 
Table 1

Characteristics of the 7 trials included in the meta-analysis.

\begin{tabular}{|c|c|c|c|c|c|c|}
\hline Trial (reference) & RAS mut $n .(\%)$ & BRAF mut $n .(\%)$ & $\begin{array}{l}\text { KRAS (mut vs wt) } \\
\text { RFS (HR, 95\% CI) }\end{array}$ & $\begin{array}{l}\text { KRAS (mut vs } \\
\text { wt) OS (HR, } \\
95 \% \mathrm{CI} \text { ) }\end{array}$ & $\begin{array}{l}\text { BRAF (mut vs wt) } \\
\text { RFS (HR,95\% CI) }\end{array}$ & $\begin{array}{l}\text { BRAF (mut vs wt) } \\
\text { OS (HR, 95\% CI) }\end{array}$ \\
\hline $\begin{array}{l}\text { Schirripa et al. } \\
\text { (2015) }\end{array}$ & $160 / 309(52)$ & $12 / 309(4)$ & $1.22(0.94-1.58)$ & $1.08(0.73-1.59)$ & $2.31(1.09-4.87)$ & $2.76(1.12-6.81)$ \\
\hline $\begin{array}{l}\text { Karagkounis et al. } \\
2013\end{array}$ & $58 / 202(29)$ & $4 / 202(2)$ & $1.78(1.04-2.71)$ & $1.97(1.20-3.24)$ & N.A & $1.90(0.60-5.99)$ \\
\hline $\begin{array}{l}\text { Kemeny et al. } \\
(2014)\end{array}$ & $51 / 169(30)$ & $1 / 162(0.6)$ & $1.90(1.12-3.20)$ & $1.99(0.88-4.51)$ & N.A & N.A \\
\hline Nasch et al. (2010) & $51 / 188(27)$ & N.A & N.A & $2.39(1.41-4.04)$ & N.A & N.A \\
\hline $\begin{array}{l}\text { Stremitzer et al. } \\
(2012)\end{array}$ & $15 / 60(25)$ & $0 / 60$ & $2.48(1.26-4.89)$ & $3.49(1.29-9.41)$ & N.A & N.A \\
\hline Teng et al. (2012) & $87 / 231(37)$ & $5 / 231(2)$ & N.A & $1.48(0.86-2.54)$ & N.A & $6.23(1.89-20.61)$ \\
\hline $\begin{array}{l}\text { Vauthey et al. } \\
\text { (2013) }\end{array}$ & $34 / 193(18)$ & N.A & $1.92(1.21-3.03)$ & $2.25(1.13-4.49)$ & N.A & N.A \\
\hline
\end{tabular}

OS indicates overall survival; RFS, relapse free survival; mut, mutated; wt, wild type; $n$., number of patients.

HR, hazard ratio; CI, confidence intervals; N.A, not available.

reach a consensus, another author was consulted and a final decision was made by consensus. Information was carefully extracted by overall selected studies. The following data were collected and organized from eligible studies: first author name, journal and year of publication, study design, study treatment, baseline characteristics of patients (i.e., age, stage, KRAS status, BRAF status), outcome measures (i.e., RFS and/or OS), and and hazard ratio (HR) with $95 \%$ confidence intervals (CIs) for RFS and/or OS, stratified according to KRAS and/or BRAF mutation status. Data extraction was conducted according to the Preferred Reporting Items for Systematic Reviews and Meta-Analysis (PRISMA) guidelines (Moher et al., 2010).

\subsection{Statistical analysis}

Patients were stratified according to KRAS and/or BRAF mutation status into 2 groups: mutant KRAS and/or BRAF and wild-type KRAS and/or BRAF. The outcome measures were OS and RFS, calculated in KRAS and/or BRAF-mutated patients undergoing resection of CLM, compared to KRAS and/or BRAF wild-type population. The correlation between these endpoints and KRAS/BRAF mutations was expressed as an HR of KRAS and/or BRAF-mutated patients over KRAS/BRAF-wild type patients. Thus, an HR more than 1 indicates that KRAS and/or BRAF-mutations are associated with worse OS and/or RFS after CLM resection. The heterogeneity between trials was tested using the Cochran $Q$-test, with a predefined significance threshold of 0.1. A meta-analysis of HRs was performed to calculate a pooled HR for each outcome using a fixed-effect or random-effect, based on statistical significance of $Q$-test, according to Mantel-Haenszel method. Publication bias for both OS and RFS analysis were assessed using Begg's funnel plots and Egger's test. The level of significance was set at $5 \%(P<0.05$ suggested a statistical significant publication bias). If publication bias was found, the Duval and Tweedie nonparametric 'trim and fill' method was used to adjust it. All statistical analyses were performed with Review Manager 5.3.5 (RevMan; version 5.3.5) and Comprehensive MetaAnalysis software (CMA; version 3.0).

\section{Results}

Our search, according to the aforementioned criteria, performed in May 2015, identified 392 publications. Among these, after a careful selection procedure, only seven studies (1403 patients) met our inclusion criteria and were included in our meta-analysis (Fig. 1). Three of these studies were retrospective series (Vauthey et al., 2013; Kemeny et al., 2014; Francesca Bergamo and Loupakis et al., 2014), while the others were prospective studies (Nash et al., 2010; Karagkounis et al., 2013; Stremitzer et al., 2012; Teng et al., 2012). In these 7 studies, sample sizes of the analyzed population ranged from 60 (Stremitzer et al., 2012) to 309 (Schirripa et al., 2015), while the percentage of KRAS mutated patients ranged from $14 \%$ (Stremitzer et al., 2012) to 37\% (Teng et al., 2012). BRAF mutations were analysed in four of the seven included studies (Karagkounis et al., 2013; Stremitzer et al., 2012; Teng et al., 2012), and it was found in $2-4 \%$ of patients who underwent surgery for CLM. In nearly every trial, patients' characteristics were well balanced between the two arms. A detailed list of the seven selected studies, including molecular genotyping and patients' outcomes, are described in Table 1.

All the included studies analyzed the survival outcomes (OS and/or RFS) of patients undergoing resection of CLM, stratified by KRAS mutation status. Five studies reported HRs with 95\% CI data for both RFS and OS (Karagkounis et al., 2013; Vauthey et al., 2013; Stremitzer et al., 2012; Kemeny et al., 2014), while two studies reported only HRs with 95\% CI data for OS (Nash et al., 2010; Teng et al., 2012). Pooled analysis showed that KRAS mutations predicted a significant worse both RFS (HR: 1.53; 95\% CI: 1.27-1.83) and OS (HR: $1.7 ; 95 \% \mathrm{CI}: 1.38-2.10$ ) in patients who underwent surgical resection of CLM (Figs. 2 and 3). The pooled HRs for both RFS and OS were calculated using fixed-effect model, because of non-significant heterogeneity between treatment effects ( $Q$-test: $P: 0.16 ; P: 0.11)$. Only three of the seven included studies analysed the OS of patients who underwent resection of CLM, according to BRAF mutation status (Karagkounis et al., 2013; Teng et al., 2012),

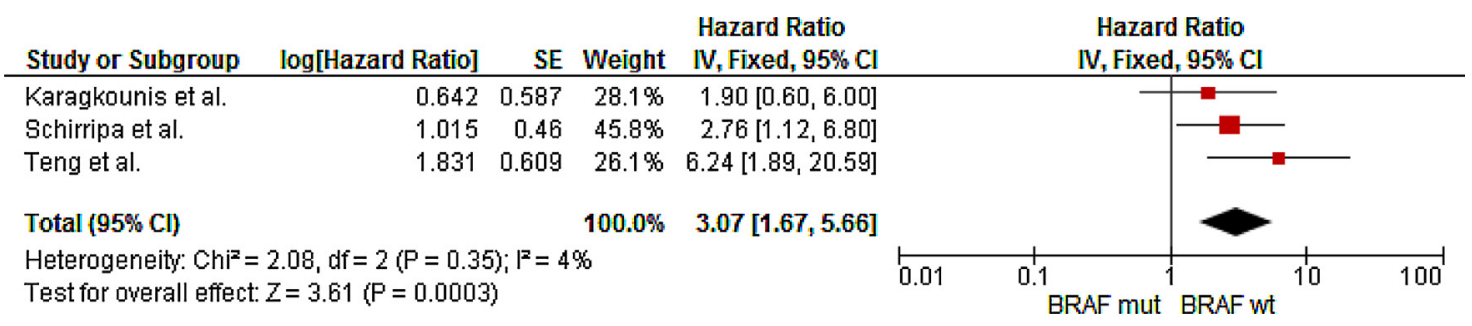

Fig. 4. Forest plot showing hazard ratio for overall survival according to BRAF mutation status in colorectal cancer patients who received resection of liver metastasis. 


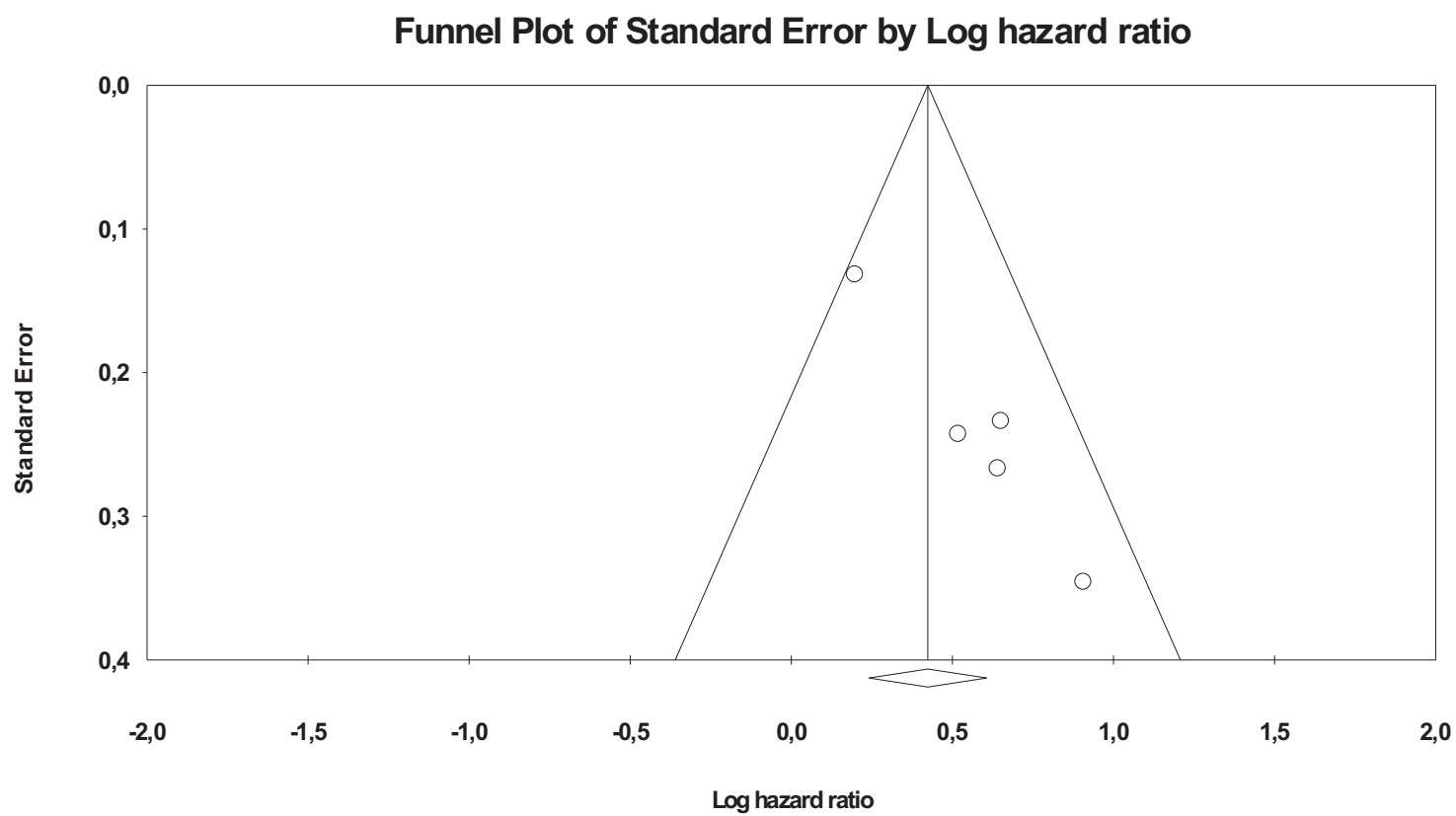

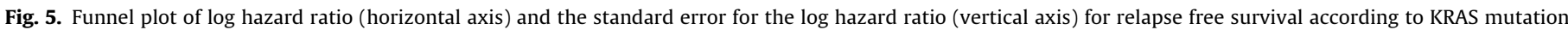

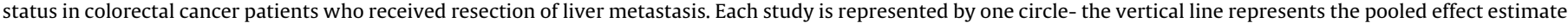

while RFS data have not been reported in any of such studies. Pooled analysis showed that BRAF mutations predicted a significant worse OS (HR: 3.07; 95\% CI: 1.67-5.66) in this subgroup of CRC patients (Fig. 4). The pooled HR for OS was calculated using fixed-effect model, because of non-significant heterogeneity between treatment effects ( $Q$-test: $P: 0.35$ ). Publication bias have not been found by Begg's test for both RFS and OS ( $P: 0.11)$, according to KRAS mutation status, while Egger's tests for both RFS and OS (HRs) analysis, resulted positive for bias ( $P: 0.0017 ; P: 0.02)$. Funnel plots for HRs of both RFS and OS stratified by KRAS mutations are reported in Figs. 5 and 6. However, analysis with the 'trim and fill' method did not draw remarkably different conclusions, confirming the sig- nificant association between KRAS mutations and worse patients' outcomes, including both OS (adjusted HR: 1.39, 95\% CI: 1.16-1.66) and RFS (adjusted HR: 1.31, 95\% CI: 1.12-1.53). Publication biases were not found for BRAF status and OS by both Begg's ( $P: 0.5)$ and Egger's tests ( $P$ : 0.39).

\section{Discussion}

This meta-analysis, including seven studies, which evaluated the survival outcomes (OS, and/or RFS) of patients undergoing hepatic resection of CLM, stratified by KRAS and/or BRAF mutation status, has shown that both KRAS and BRAF mutations are asso-

Funnel Plot of Standard Error by Log hazard ratio

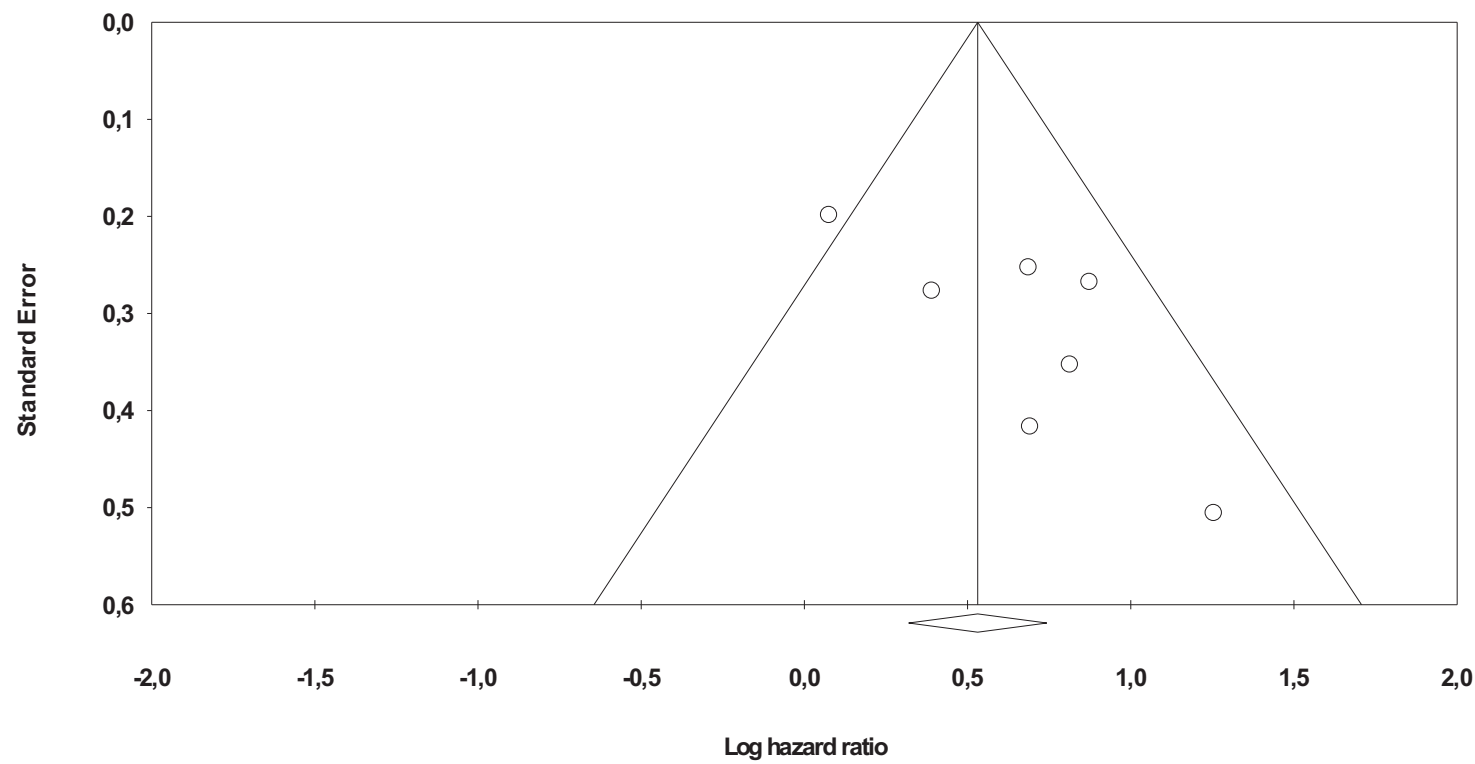

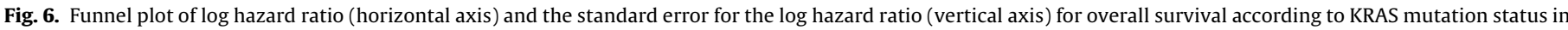
colorectal cancer patients who received resection of liver metastasis. Each study is represented by one circle- the vertical line represents the pooled effect estimate. 
ciated with worse patients' survival outcomes, compared to KRAS and BRAF wild-type population. Indeed both the risk of recurrence and death are significantly higher in both KRAS and BRAF mutated compared to KRAS and BRAF wild-type patients, suggesting a negative prognostic value of such molecular biomarkers in this subgroup of patients. Currently, few clinical-pathological factors are considered to predict the recurrence risk and outcomes of patients undergoing resection of CLM in clinical practice, but our ability to identify patients who may benefit more from surgical therapies still very poor, and very little is known about the biology underlying the tumor heterogeneity. The role of molecular biomarkers, such as KRAS and BRAF, is well established in the advanced and unresectable disease, but its potential application in CRC patients with liver metastases candidate to resection has not been defined yet. The results of our work suggest both KRAS and BRAF mutations as negative prognostic biomarkers in patients undergoing surgical therapy for CLM, supporting the evaluation of such molecular biomarkers together with the other clinical-pathological factors, in order to more accurately predict, both recurrence risk and survival of patients undergoing resection of CLM, and ultimately select only the best candidates for surgery. Indeed it's possible that both KRAS and BRAF mutations are associated with a worse biology and a more rapid and aggressive metastatic behavior of CLM, discouraging surgeons to perform surgical resection, independently of clinical resectability. This interpretation is consistent with the evidence that the percentage of KRAS mutated patients ranged from 14\% (Stremitzer et al., 2012) to 37\% (Teng et al., 2012) in all seven included studies, compared to $35-45 \%$ reported in metastatic and unresectable CRC, suggesting a significant association between KRAS mutations rates and tumor behavior, as described in preclinical studies (Suchy et al., 1992; Tanaka et al., 1994; Varghese et al., 2002; Webb et al., 1998). Some clinical series have also shown that KRAS mutations are associated with an increased risk of developing lung and liver metastases compared to wild-type population (Vauthey et al., 2013; Kemeny et al., 2014). Others have shown a higher incidence of lung metastases/recurrence than liver metastases/recurrence in KRAS mutated patients (Vauthey et al., 2013; Tie et al., 2011; Kim et al., 2012). Even if KRAS mutations rate in CLM usually corresponds with the mutation status of the primary tumor (Tie et al., 2011; Vakiani et al., 2012), it seems to be different among different metastatic sites, with a higher percentage of mutations in lung and brain metastases, than in liver metastases (Tie et al., 2011; Kim et al., 2012), suggesting specific RAS-related patterns of recurrence, with potential implication in the clinical management of CRC patients, but these trends need to be investigated in larger, prospective studies. As regards BRAF mutations, even if evaluated in a small subgroup of patients, our findings are consistent with the most part of published studies which have validated BRAF mutations obtained from primary tumour specimens as a strong negative prognostic biomarker in metastatic CRC (Ahn et al., 2014), showing a more aggressive and chemo-refractory behavior compared to wild-type tumours (Tran et al., 2011). However it's interesting to observe that the percentages of BRAF mutations $(2-4 \%)$ in all the included studies is lower than those reported in the literature (5-10\%) (Sridhar et al., 2005; Di Nicolantonio et al., 2008), likely due to the clinical selection of patients included in such studies. Indeed, because of their peculiar metastatic spread, BRAF-mutated tumours are usually detected as an advanced disease, rarely candidate for liver surgery. The low percentage of BRAF mutations in such subgroup of CRC patients, could limit its clinical application as prognostic biomarker for routinary use, but does not affect neither its scientific relevance, nor its potential implications for individual patients.

This literature-based meta-analysis confirms the results achieved in the majority of included studies, suggesting both KRAS and BRAF mutations as, negative, prognostic biomarker, in patients who underwent surgical resection of CLM; however, it has some limitations. The patients cohorts included in the seven studies of our meta-analysis are quite heterogeneous, because of different selection criteria, as well as a variable origin (primary tumor vs liver metastases) of tissue available for the mutational analysis. Indeed the mutation status was determined from liver metastasis for the majority of patients, while it was determined from the primary tumor in about $20 \%$ of patients included in both the Kemeny et al. (2014) and Schirripa et al. (2015) studies. Almost all seven studies included, performed the evaluation of the most common KRAS mutations in the extracted DNA (codon12/13); only two studies (Vauthey et al., 2013; Schirripa et al., 2015) evaluated both KRAS and NRAS mutations (codon12/13/61), stratifying patients according to all RAS mutation status. However, NRAS mutations account for only $10-20 \%$ of all RAS-mutated patients, representing a very small subgroup of patients included in both studies, likely not influencing final study results. Almost all the patients included in our meta-analysis had received peri-operative, 5-FU-based, systemic chemotherapy, with or without bevacizumab. Only a small percentage of patients had received anti-EGFR MoAbs $(11.8 \%$ in the study of Teng et al. (2012), 5\% in the study of Schirripa et al. (2015), and 13 of 28 wild-type KRAS patients who developed recurrence, in the study of Stremitzer et al. (2012)). Furthermore two of the seven studies, included also patients who received locoregional treatments after liver resection, such as adjuvant hepatic arterial infusion (HAI) (Kemeny et al., 2014), and tumour ablation (Karagkounis et al., 2013). The above described heterogeneity among the different patients' cohort, does not reduce the clinical and statistical significance of our results, which reveal both KRAS and BRAF as poor prognostic biomarker in patients who underwent surgical resection of CLM, but limits, of course, any generalization to the overall CRC population, as well as its current use as predominant factor for the upfront patients selection in clinical practice. Indeed, the lack of a larger individual patient analysis, including also the other known clinical-pathological prognostic factors, such as the number and size of CLM, the disease-free interval from primary to CLM, CEA level, primary tumor stage, etc., does not allow to consider both KRAS and BRAF mutations as independent prognostic biomarkers for clinical decision models. Finally, longterm survival outcomes (10-20 year RFS/OS rate) should be known before to definitively exclude CRC patients from surgery, but such data were not reported in any of included studies. Therefore further clinical trials are urgently needed to investigate if KRAS/BRAF mutated patients undergoing hepatectomy for CLM, have significantly lower RFS/OS rates compared to the wild type, after a long-term follow-up, and ultimately recommend, as suggested by our work, the surgical treatment of CLM only to the wild type population. In conclusion our meta-analysis confirm both KRAS and BRAF mutations as a strong, prognostic biomarkers, associated with worse both OS and RFS, in patients undergoing hepatic resection of CLM. Certainly, these interesting evidences represent the first step towards a deeper understanding of molecular mechanisms underlying the tumor behavior and patients' outcomes in a clinicalselected subgroup of CRC patients, supporting the introduction of new treatment decision models, taking into account the tumor molecular profile, together with the other clinical-pathological factors, in order to predict the outcomes of patients undergoing resection of CLM, and individualize both systemic and loco-regional treatment strategies.

\section{Conflict of interest}

The authors state no conflict of interest and have received no payment in the preparation of this manuscript. 


\section{References}

Adam, R., Hoti, E., Folprecht, G., Benson, A.B., 2009. Accomplishments in 2008 in the management of curable metastatic colorectal cancer. Gastrointest. Cancer Res. 3, S15-S22.

Ahn, T.S., et al., 2014. The BRAF mutation is associated with the prognosis in colorectal cancer. J. Cancer Res. Clin. Oncol. 140, 1863-1871.

Bokemeyer, C., et al., 2012. Addition of cetuximab to chemotherapy as first-line treatment for KRAS wild-type metastatic colorectal cancer: pooled analysis of the CRYSTAL and OPUS randomised clinical trials. Eur. J. Cancer 48, 1466-1475.

Bokemeyer, C., et al., 2015. FOLFOX4 plus cetuximab treatment and RAS mutations in colorectal cancer. Eur. J. Cancer.

Bronte, G., et al., 2011. EGFR genomic alterations in cancer: prognostic and predictive values. Front. Biosci. (Elite Ed.) 3, 879-887.

Bronte, G., Rolfo, C., Peeters, M., Russo, A., 2014. How to find the Ariadne's thread in the labyrinth of salvage treatment options for metastatic colorectal cancer? Expert Opin. Biol. Ther. 14, 743-748.

Bronte, G., et al., 2013. Monoclonal antibodies in gastrointestinal cancers. Expert Opin. Biol. Ther. 13, 889-900.

Bronte, G., et al., 2015. Monoclonal antibodies for the treatment of non-haematological tumours: update of an expanding scenario. Expert Opin. Biol. Ther. 15, 45-59

Chiappa, A., et al., 2009. The management of colorectal liver metastases: Expanding the role of hepatic resection in the age of multimodal therapy. Crit Rev. Oncol. Hematol. 72, 65-75.

Choti, M.A., et al., 2002. Trends in long-term survival following liver resection for hepatic colorectal metastases. Ann. Surg. 235, 759-766.

Chua, T.C., Saxena, A., Chu, F., Zhao, J., Morris, D.L., 2011. Predictors of cure after hepatic resection of colorectal liver metastases: an analysis of actual 5- and 10-year survivors. J. Surg. Oncol. 103, 796-800.

De Roock, W., et al., 2010. Effects of KRAS, BRAF, NRAS, and PIK3CA mutations on the efficacy of cetuximab plus chemotherapy in chemotherapy-refractory metastatic colorectal cancer: a retrospective consortium analysis. Lancet Oncol. 11, 753-762.

Di Nicolantonio, F., et al., 2008. Wild-type BRAF is required for response to panitumumab or cetuximab in metastatic colorectal cancer. J. Clin. Oncol. 26, 5705-5712.

Douillard, J.Y., et al., 2013. Panitumumab-FOLFOX4 treatment and RAS mutations in colorectal cancer. N. Engl. J. Med. 369, 1023-1034.

Fong, Y., Fortner, J., Sun, R.L., Brennan, M.F., Blumgart, L.H., 1999. Clinical score for predicting recurrence after hepatic resection for metastatic colorectal cancer: analysis of 1001 consecutive cases. Ann. Surg. 230, 309-318 (discussion 318-321).

Francesca Bergamo, M.S., Loupakis, Fotios, et al., 2014. J. Clin. Oncol. 34 (Suppl. 3 ) (abstr 476).

Garden, O.J., et al., 2006. Guidelines for resection of colorectal cancer liver metastases. Gut 55 (Suppl. 3), iii1-iii8.

Hecht, J.R., et al., 2009. A randomized phase IIIB trial of chemotherapy, bevacizumab, and panitumumab compared with chemotherapy and bevacizumab alone for metastatic colorectal cancer. J. Clin. Oncol. 27, 672-680.

Heinemann, V., Von Weikersthal, L.F., Decker, T., et al., 2014. FOLFIRI plus cetuximab versus FOLFIRI plus bevacizumab as first-line treatment for patients with metastatic colorectal cancer (FIRE-3): a randomised, open-label, phase 3 trial. Lancet Oncol. 15 (September (10)), 1065-1075.

Karagkounis, G., et al., 2013. Incidence and prognostic impact of KRAS and BRAF mutation in patients undergoing liver surgery for colorectal metastases. Cancer 119, 4137-4144.

Kastrinakis, W.V., Ramchurren, N., Maggard, M., Steele, G., Summerhayes, I.C., 1995. K-ras status does not predict successful hepatic resection of colorectal cancer metastasis. Arch. Surg. 130, 9-14.

Kemeny, N.E., et al., 2014. KRAS mutation influences recurrence patterns in patients undergoing hepatic resection of colorectal metastases. Cancer 120, 3965-3971.

Kim, M.J., et al., 2012. Different metastatic pattern according to the KRAS mutational status and site-specific discordance of KRAS status in patients with colorectal cancer. BMC Cancer 12, 347.

Kopetz, S., et al., 2009. Improved survival in metastatic colorectal cancer is associated with adoption of hepatic resection and improved chemotherapy. J. Clin. Oncol. 27, 3677-3683.

Ksienski, D., Woods, R., Speers, C., Kennecke, H., 2010. Patterns of referral and resection among patients with liver-only metastatic colorectal cancer (MCRC). Ann. Surg. Oncol. 17, 3085-3093.

Mann, C.D., Metcalfe, M.S., Leopardi, L.N., Maddern, G.J., 2004. The clinical risk score: emerging as a reliable preoperative prognostic index in hepatectomy for colorectal metastases. Arch. Surg. 139, 1168-1172.

Maughan, T.S., et al., 2011. Addition of cetuximab to oxaliplatin-based first-line combination chemotherapy for treatment of advanced colorectal cancer: results of the randomised phase 3 MRC COIN trial. Lancet 377, 2103-2114

Moher, D., Liberati, A., Tetzlaff, J., Altman, D.G., Group, P., 2010. Preferred reporting items for systematic reviews and meta-analyses: the PRISMA statement. Int. J. Surg. 8, 336-341.

Nagashima, I., Takada, T., Nagawa, H., Muto, T., Okinaga, K., 2006a. Proposal of a new and simple staging system of colorectal liver metastasis. World J. Gastroenterol. 12, 6961-6965.
Nagashima, I., et al., 2006b. Proposal of criteria to select candidates with colorectal liver metastases for hepatic resection: comparison of our scoring system to the positive number of risk factors. World J. Gastroenterol. 12, 6305-6309.

Nash, G.M., et al., 2010. KRAS mutation correlates with accelerated metastatic progression in patients with colorectal liver metastases. Ann. Surg. Oncol. 17, $572-578$

Nordlinger, B., et al., 1996. Surgical resection of colorectal carcinoma metastases to the liver. A prognostic scoring system to improve case selection, based on 1568 patients. Association Française de Chirurgie. Cancer 77, 1254-1262.

Price, T.J., et al., 2011. Impact of KRAS and BRAF gene mutation status on outcomes from the phase III AGITG MAX trial of Capecitabine alone or in combination with Bevacizumab and Mitomycin in advanced colorectal cancer. J. Clin. Oncol. 29, 2675-2682.

Richman, S.D., et al., 2009. KRAS and BRAF mutations in advanced colorectal cancer are associated with poor prognosis but do not preclude benefit from oxaliplatin or irinotecan: results from the MRC FOCUS trial. J. Clin. Oncol. 27, 5931-5937.

Rizzo, S., et al., 2010. Prognostic vs predictive molecular biomarkers in colorectal cancer: is KRAS and BRAF wild type status required for anti-EGFR therapy? Cancer Treat. Rev. 36 (Suppl. 3), S56-S61.

Rolfo, C., Russo, A., Santini, D., Bronte, G., Peeters, M., 2013. Dilemma in metastatic colorectal cancer: VEGF versus EGRF targeting. Expert Opin. Ther. Targets 17, 869-871

Rolfo, C., et al., 2014. The role of targeted therapy for gastrointestinal tumors Expert Rev. Gastroenterol. Hepatol. 8, 875-885.

Russo, A., et al., 1998. Prognostic significance of proliferative activity, DNA-ploidy, p53 and Ki-ras point mutations in colorectal liver metastases. Cell Prolif. 31, $139-153$.

Russo, A., et al., 2009. The long and winding road to useful predictive factors for anti-EGFR therapy in metastatic colorectal carcinoma: the KRAS/BRAF pathway. Oncology 77 (Suppl. 1), 57-68.

Schirripa, M., et al., 2015. BRAF and RAS mutations as prognostic factors in metastatic colorectal cancer patients undergoing liver resection. $\mathrm{Br}$. J. Cancer.

Schwartzberg, L.S., et al., 2014. PEAK: a randomized, multicenter phase II study of panitumumab plus modified fluorouracil, leucovorin, and oxaliplatin (mFOLFOX6) or bevacizumab plus mFOLFOX6 in patients with previously untreated, unresectable, wild-type KRAS exon 2 metastatic colorectal cancer. J. Clin. Oncol. 32, 2240-2247.

Sridhar, S.S., Hedley, D., Siu, L.L., 2005. Raf kinase as a target for anticancer therapeutics. Mol. Cancer Ther. 4, 677-685.

Stremitzer, S., et al., 2012. KRAS status and outcome of liver resection after neoadjuvant chemotherapy including bevacizumab. Br. J. Surg. 99, 1575-1582.

Suchy, B., Zietz, C., Rabes, H.M., 1992. K-ras point mutations in human colorectal carcinomas: relation to aneuploidy and metastasis. Int. J. Cancer 52, 30-33.

Tanaka, M., Omura, K., Watanabe, Y., Oda, Y., Nakanishi, I., 1994. Prognostic factors of colorectal cancer: K-ras mutation, overexpression of the p53 protein, and cell proliferative activity. J. Surg. Oncol. 57, 57-64

Taylor, A., Kanas, G., LW, et al., 2010. Ann. Oncol. 21 (Suppl. 8), 632P.

Tejpar, S., et al., 2012. Association of KRAS G13D tumor mutations with outcome in patients with metastatic colorectal cancer treated with first-line chemotherapy with or without cetuximab. J. Clin. Oncol. 30, 3570-3577.

Teng, H.W., et al., 2012. BRAF mutation is a prognostic biomarker for colorectal liver metastasectomy. J. Surg. Oncol. 106, 123-129.

Tie, J., et al., 2011. KRAS mutation is associated with lung metastasis in patients with curatively resected colorectal cancer. Clin. Cancer Res. 17, 1122-1130.

Tol, J., et al., 2010. Markers for EGFR pathway activation as predictor of outcome in metastatic colorectal cancer patients treated with or without cetuximab. Eur. J Cancer 46, 1997-2009.

Tran, B., et al., 2011. Impact of BRAF mutation and microsatellite instability on the pattern of metastatic spread and prognosis in metastatic colorectal cancer. Cancer 117, 4623-4632

Vakiani, E., et al., 2012. Comparative genomic analysis of primary versus metastatic colorectal carcinomas. J. Clin. Oncol. 30, 2956-2962.

Van Cutsem, E., et al., 2015. Fluorouracil, leucovorin, and irinotecan plus cetuximab treatment and RAS mutations in colorectal cancer. J. Clin. Oncol. 33, 692-700.

Varghese, H.J., et al., 2002. Activated ras regulates the proliferation/apoptosis balance and early survival of developing micrometastases. Cancer Res. 62, 887-891.

Vauthey, J.N., et al., 2013. RAS mutation status predicts survival and patterns of recurrence in patients undergoing hepatectomy for colorectal liver metastases. Ann. Surg. 258, 617-626.

Webb, C.P., Van Aelst, L., Wigler, M.H., Vande Woude, G.F., 1998. Signaling pathways in Ras-mediated tumorigenicity and metastasis. Proc. Natl. Acad. Sci. U. S. A. 95, 8773-8778.

de Jong, M.C., et al., 2009. Rates and patterns of recurrence following curative intent surgery for colorectal liver metastasis: an international multi-institutional analysis of 1669 patients. Ann. Surg. 250, 440-448.

\section{Biographies}

Francesco Passiglia, M.D., received his M.D. degree from University Medical School of Palermo, Italy, in 2011. He's attending residency school in Medical Oncology at the same University. He is interested in the field of Non-Small Cell Lung Cancer and Colorectal Cancer treatment with optimization of targeted drug tolerability. He works at the University Hospital of Palermo and collaborates with other cancer cen- 
tres in Europe. He spent 8 months at the Phase I- Early Clinical Trials Unit, Oncology Department, Antwerp University Hospital Belgium. He is author of more than 20 publications in top-rated international cancer journals.

Giuseppe Bronte, M.D., Ph.D., received his M.D. degree from University Medical School of Palermo (Italy) in 2004. His post-graduate specialty was in Medical Oncology in 2008. He received his PhD degree in Experimental and Clinical Oncology from the same University in 2012. Co-investigator and Data manager in Multicenter Clinical Trials, managed by different Clinical Research Cooperative Groups, according to Good Clinical Practice (ITMO, SICOG, GOIM). He is a member of scientific societies and he is actively involved in the teaching and research of oncology fellows and students. He is author more than 40 publications in top-rated international cancer journals.

Viviana Bazan, Ph.D., is Associate Professor at University Medical School of Palermo, Department of Experimental Biomedicin and Clinical Neuroscience (Italy). From 2008 to 2010, she has been an Adjunct Associate Professor at Temple University's College of Science and Technology, Philadelphia (USA). She has been Co-Editor of Annals of Oncology (Volume 17, 2006 and Volume 18, 2007). Over the last few years, she has been implicated in clinical oncology research aimed at identifying biomolecular prognostic features and treatment response. In this context she has been concerned with the molecular genetics of sporadic, hereditary and familial tumors. She is the author of more than 80 publications in top-rated cancer journals.

Antonio Galvano, M.D., received his M.D. degree from University Medical Schoo of Palermo, Italy, in 2010. He is attending residency school in Medical Oncology at the same university. He actually works at University of Palermo focusing his oncology interest in studying molecular mechanisms and targeted drugs in Gastrointestina and Lung neoplasms. He spent 7 months at "G.B. Rossi" Hospital, Department of Oncology of the University of Verona and is sub-investigator in many of clinica trials active in his centre.

Bruno Vincenzi, M.D., Ph.D., was born in Velletri on July 16th, 1976. He is a medical oncologist in the Medical Oncology Department of Campus Bio-Medico University of Rome and Associate Professor in Medical Oncology in the same University.
He is the author of about 300 papers published in international peer-reviewed journals. He is actively involved in clinical and translational research. Current fields of investigation are addressed to the clinical and molecular aspects of solid tumors, to the study of angiogenesis in cancer and to the development of antiangiogenetic drugs. He is recognized as an international expert in the treatment of gastrointestinal cancers and soft tissue sarcomas.

Antonio Russo, M.D., Ph.D., is Professor of Medical Oncology at University Medical School of Palermo, Department of Surgical, Oncological and Stomatologica Sciences (Italy). From 2004 to July 2011 he has been an Adjunct Associate Professor and since August 2011 Adjunct Full Professor at Temple University's College of Science and Technology, Philadelphia (USA). Since February 2012 is Director of Medica Oncology Unit and Director of Regional Reference Center for Prevention, Diagnosis and Treatment of Rare Tumors and Heredo-familial Solid Tumors in Adults, AOUP "P. Giaccone", Palermo (Italy). Since April 2012 is Director of the Specialization School in Medical Oncology, University of Palermo, School of Medicine, Palermo, Italy. Since November 2013 Medical Oncology Unit directed by Prof A Russo has been recognized as a 2013 ESMO Designated Centres of Integrated Oncology and Palliative Care.Since 2001 he has been a coordinator with Prof D. Kerr (University of Oxford, UK) and Prof B. Iacopetta (Western Australia University) of the "CRCP53 International Collaborative Study". Since 2003 he has been an expert member of INSERM (Institut National de la Santè et de la Recherche Mèdical, France), since 2007 of Scientific Committee INCA (Institut National du Cancer, France) and of NWCRF (North West Cancer Research Fund, UK). He is member of Editorial board of Journal of Carcinogenesis \& Mutagenesis (since 2011) and World Journal of Gastrointestinal Oncology and World Journal of Clinical Oncology (since 2012). Since 2013 is Associate Editor of Journal of Solid Tumors. Since 2008 he has been Guest Editor of Annals of Oncology $(2006,2007)$. The central theme of his studies is translational research, meaning the application of molecular genetics in cancer management. He is PI in several national and international clinical trials. He is the author of more than 300 peer-reviewed publications listed on Medline-PubMed. 